\title{
MODELLING AIDS EPIDEMIC AND TREATMENT WITH DIFFERENCE EQUATIONS
}

\author{
K. M. TAMIZHMANI, A. RAMANI, B. GRAMMATICOS, AND A. S. CARSTEA
}

Received 15 October 2003 and in revised form 10 March 2004

We propose two models for the description of the dynamics of an AIDS epidemic and of the effect of a combined-drugs AIDS treatment based on difference equations. We show that our interacting population model, despite its extreme simplicity, describes adequately the evolution of an AIDS epidemic. A cellular-automaton analogue of the discrete system of equations is presented as well. In the case of drug treatment, we identify two different regimes corresponding to efficient and inefficient medication. The effect of the discreteness of the equations is also studied.

\section{Introduction}

The modelling of biological systems goes back in time to an era when the very word "modelling," in the present acceptation, was unknown. The use of mathematical modelling, which has met with such a great success in physics, was extended to the description of the behavior of living organisms under various conditions. The advantage of this "paperware," rather than "wetware," laboratory approach is clear. Mathematical models allow us to explore the effect of changes of various parameters in biological systems in an easy, fast, and inexpensive way, while the real experiment may be sometimes unfeasible (to say nothing of the ethical issues). Moreover, the explicit construction of the mathematical model constrains the modeller to a detailed analysis of the mechanisms involved, which leads to a better understanding of the whole process.

The mathematical models [8] of biological processes can be classified into two broad categories: stochastic and deterministic models. In the first, one is interested in the behavior of small samples, where fluctuations can play an important role and probabilistic answers are usually sought. In the second, one deals with larger samples and the model is usually expressed in terms of differential equations [13]. In what follows, we will present models based on difference rather than differential equations. While we will eschew the discussion of the true nature of time, we must point out that discrete systems are more fundamental than continuous ones. They contain the latter as special limits and, moreover, possess other limits besides the continuous one. From a practical point of view, and 
in particular for biological systems, bookkeeping constraints, like population counting, introduce a discreteness in the data gathering which must, in principle, be reflected in the model's equations. Moreover, the various processes involved have characteristic times which enhance the necessity for a discrete-time treatment. The only delicate point is the choice of an optimal time step. Another advantage of discrete systems is that they are "natural" integrators of the corresponding differential systems. Their use is preferable over that of numerical schemes which can be unstable or cumbersome and, in any case, black-box-like and do not offer any insight into the process. The main question is how to obtain an adequate discretization of a continuous system. The fact that infinitely many discrete systems may have the same continuous limit only complicates the matter further. Our approach consists in preserving as many of the properties of the continuous system as possible.

In the preceding paragraph, we alluded to special limits of discrete systems different from the continuous ones. A most interesting such limit is the one known under the name of ultradiscrete limit. Introduced by the Tokyo-Kyoto group, this limit allows one, given a discrete system, to obtain a generalized cellular automaton [12]. The importance of such systems in modelling is capital: it suffices to point out that all numerical simulations with finite-precision arithmetic are, in fact, based on generalized cellular automata. The advantage of the ultradiscretization procedure is that it is a systematic, algorithmic approach which, moreover, generates systems that encapsulate the essential, albeit in a bare-bones version, dynamical behavior of the initial system.

In what follows, we will present two models which are related to AIDS. The first is an extremely simple model for the description of the onset and evolution of an AIDS epidemic. The second studies the effect of a combined treatment on the population of Tcells, the latter being an indicator of the appearance of AIDS in seropositive individuals.

\section{A discrete SIR-like model for AIDS epidemics}

In this section, we will present a simple model for the description of the dynamics of an AIDS epidemic. Our aim is to make the model as simple as possible and still be able to describe correctly the dynamics [1]. Our starting point is the so-called SIR model. Introduced several decades ago by Kermack and McKendrick [3, 4, 5], this model is based on the splitting of the population into three interacting classes: the susceptible, a priori healthy individuals, the infected ones who are also infective, and the removed who, having contracted the infection, are now cured and immune (or dead).

The variant of this model that we will use below is the following SIA model. We also consider a population consisting of three classes. The first class is again that of healthy, susceptible individuals (S). The second class (I) is that of HIV-positive individuals who, interacting with the susceptible ones, can infect them. The third class (A) is that of the AIDS patients (who we assume not to be infective). It is clear that more sophisticated models can be and have been proposed, which correspond to a finer splitting of the population into interacting classes. More simplifying assumptions will be introduced in order to build up the model. They are not essential and could be very easily relaxed. Still, we feel that it is interesting to simplify the model as much as we can and still have it describe correctly the process of an AIDS epidemic. Thus, we make the assumption that the 
population of susceptible individuals is initially fixed and can only decrease. Next, we neglect the effect of natural death on all three populations: individuals get removed only at the AIDS stage. We start by writing the differential equations which describe this model:

$$
\frac{d S}{d t}=-S I, \quad \frac{d I}{d t}=S I-\mu I, \quad \frac{d A}{d t}=\mu I-\lambda A,
$$

where we have normalized the strength of the interaction term $S I$ to 1 through a proper redefinition of time. Moreover, $\lambda>\mu(>0)$ since the evolution towards the final demise is faster than that of seropositivity towards AIDS. We remark readily that the model is such that $(S+I)^{\prime}=-\mu I$ (where the prime denotes the time derivative) and $(S+I+A)^{\prime}=-\lambda A$, that is, the population of the non-AIDS individuals decreases at a rate proportional to the infectives, while the total population decrease rate is proportional to the number of AIDS patients.

In $[11,13]$, we have presented discrete versions of SIR models. The same basic approach will be used here. Starting with the equation $S^{\prime}=-S I$, we propose the discrete analogue $x_{n+1}=x_{n} /\left(1+y_{n}\right)$. It is easy to show that if we put $x_{n}=\epsilon S, y_{n}=\epsilon I$ and take $t=\epsilon n$ at the limit $\epsilon \rightarrow 0$, the difference equation goes over to the differential one. For the discretization of the remaining equations, the main guides are the two population constraints: $(S+I)^{\prime}=-\mu I,(S+I+A)^{\prime}=-\lambda A$. It is indeed straightforward to show that the system

$$
x_{n+1}=\frac{x_{n}}{1+y_{n}}, \quad y_{n+1}=\alpha y_{n}+\frac{x_{n} y_{n}}{1+y_{n}}, \quad z_{n+1}=(1-\alpha) y_{n}+\beta z_{n}
$$

has (2.1) as the continuous limit, with $z_{n}=\epsilon A$. Moreover, at a difference equation level, it does indeed satisfy the two population constraints $\left(x_{n+1}+y_{n+1}\right)-\left(x_{n}+y_{n}\right) \propto y_{n}$ and $\left(x_{n+1}+y_{n+1}+z_{n+1}\right)-\left(x_{n}+y_{n}+z_{n}\right) \propto z_{n}$. For the two parameters, we have $\beta<\alpha<1$, which corresponds to the fact that $\lambda>\mu>0$ at the continuous limit. A characteristic of this model is that it predicts an unavoidable AIDS epidemic. Indeed, from (2.1), and mutatis mutandis also from (2.2), if we start, as is reasonable, with $A=0$, we have $A^{\prime}>0$, and thus $A$ grows as long as $A<\mu I / \lambda$. The only fixed point of (2.1) is $A=I=0$, that is, the process stops when all the infected are removed, but $S$ is free and can only be determined through a detailed evolution. Exactly the same conclusions apply to the discrete system (2.2).

One remark is in order at this point concerning the discrete system. While the continuous equations (2.1) can be evolved either forward or backward in time, this does not look possible for the discrete system because of the equation for $y_{n+1}$. Indeed, if we try to compute $y_{n}$ in terms of $y_{n+1}$, we obtain the quadratic equation $\alpha y_{n}^{2}+\left(x_{n}+\alpha-y_{n+1}\right) y_{n}-$ $y_{n+1}=0$. Thus, at each step, we have two solutions for $y_{n}$ and, a priori, a proliferating number of possible paths. However, this is not the case here. A close look at the solutions of the quadratic equation shows that they are of opposite signs. Thus, it suffices, at each step, to discard the negative solution and keep only the physical one, $y_{n}>0$.

Figures 2.1 and 2.2 show a typical evolution of the discrete model (2.2). The onset and evolution of AIDS epidemic are clearly depicted. Moreover, while the total population of susceptibles is affected, the situation does not evolve towards a pandemic where the 


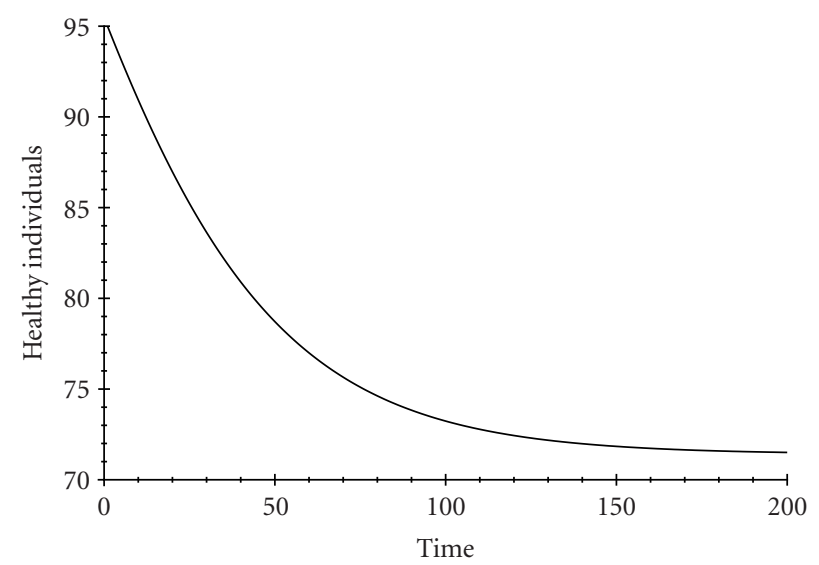

Figure 2.1. Population of susceptible (healthy) individuals as a function of time.

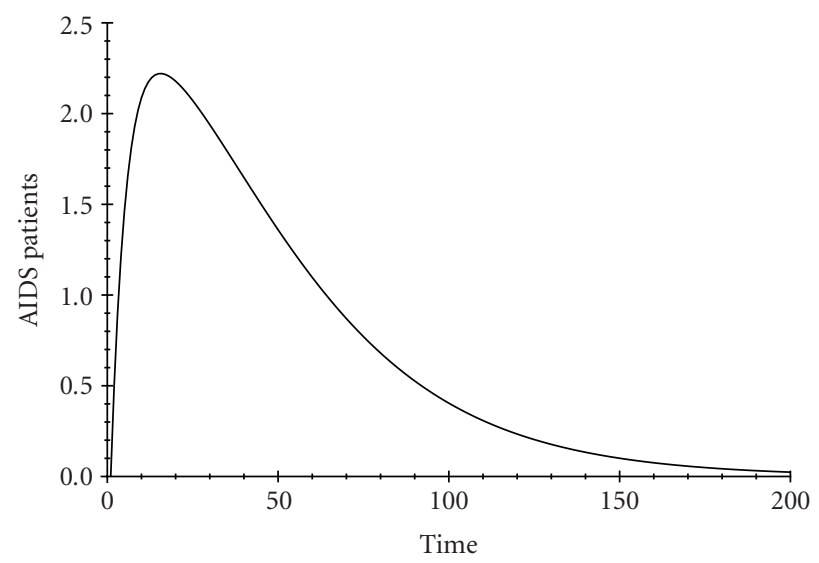

Figure 2.2. Population of AIDS patients as a function of time.

quasi-total population perishes. This last statement depends of course on the parameters involved: one can imagine situations where a pandemic erupts through an appropriate fine-tuning of the parameters.

Having established the pertinence of the discrete model, we proceed now to the derivation of the cellular-automaton analogue through ultradiscretization. The procedure we will follow is a well-established one [12]. In order to obtain the ultradiscrete limit, we start with an equation for $x$, introduce $X$ through $x=e^{X / \epsilon}$, and then appropriately take the limit $\epsilon \rightarrow 0$. Clearly, the substitution $x=e^{X / \epsilon}$ requires $x$ to be positive. The key relation is the following limit:

$$
\lim _{\epsilon \rightarrow 0^{+}} \epsilon \log \left(1+e^{x / \epsilon}\right)=\max (0, x)=\frac{x+|x|}{2}
$$


It is easy to show that $\lim _{\epsilon \rightarrow 0^{+}} \epsilon \log \left(e^{x / \epsilon}+e^{y / \epsilon}\right)=\max (x, y)$, and the extension to $n$ terms in the argument of the logarithm is straightforward.

In order to apply this procedure to our equations, we put $x=e^{X / \epsilon}, y=e^{Y / \epsilon}, z=e^{Z / \epsilon}$, $\alpha=e^{-A / \epsilon}, \beta=e^{-B / \epsilon}$, and $1-\alpha=e^{-\tilde{A} / \epsilon}$, so that $A, B, \tilde{A}>0$, and finally obtain

$$
\begin{gathered}
X_{n+1}=X_{n}-\max \left(0, Y_{n}\right), \\
Y_{n+1}=\max \left(Y_{n}-A, X_{n}+Y_{n}-\max \left(0, Y_{n}\right)\right), \\
Z_{n+1}=\max \left(Y_{n}-\tilde{A}, Z_{n}-B\right) .
\end{gathered}
$$

Thus, equation (2.4) indeed represents a generalized cellular automaton: if the parameters $A, B, \tilde{A}$ and the initial conditions $X_{0}, Y_{0}, Z_{0}$ are integers, the evolution of (2.4) will produce only integer results. Of course, the variables $X, Y, Z$ are no more positive-definite, since they are related to the logarithms of the initial variables $x, y, z$.

Since the evolution defined by the equations of system (2.4) involves piecewise linear equations and the max function, one can describe the dynamics of the ultradiscrete system in a precise, exact way by introducing the appropriate domains. By this we mean that the first equation of the system can be written as $X_{n+1}=X_{n}-Y_{n}$ when $Y_{n}>0$, and $X_{n+1}=X_{n}$ when $Y_{n}<0$, and so on. Instead of going through this analysis, which, although interesting from a rigorous point of view, does not add much to the understanding of the AIDS-epidemic model, we prefer to present an explicit simulation using (2.4). We choose $A=10, \tilde{A}=3, B=2$ and start from initial conditions $X_{0}=10, Y_{0}=5, Z_{0}=-1$. We find the following evolution:

$$
\begin{aligned}
& (10,5,-1),(5,10,2),(-5,5,7),(-10,-5,5), \\
& (-10,-15,2),(-10,-25,0),(-10,-35,-2), \ldots
\end{aligned}
$$

We remark that $X$ decreases and reaches its asymptotic value in a finite number of time steps, while $Y, Z$, after an initial increase (onset of the epidemic), start decreasing and continue towards $-\infty$.

\section{A discrete model for combined-drugs AIDS therapy}

In this section, we will present a model which deals with AIDS at a more "microscopic" level [9]. Namely, we will consider populations of viruses and of lymphocytes known as CD4 T-cells (which are the main targets of the HIV) under the influence of drugs.

The dynamics of the HIV infection have been well established by now [2]. Upon infection, the number of HIV in the blood increases sharply but then declines (over a period of a few months) to much lower levels at which it can stay with very small variations for several years till the appearance of full-blown AIDS, which is accompanied again by an increase. The T-cell count after an initial fast decrease stabilizes at levels which are lower than those of healthy individuals but not alarmingly so. Finally, a fast drop of the T-cell count can be used as a criterion to diagnose the actual onset of AIDS. During the long "dormant" period of the virus, the immune response of the organism leads to a constant production of antibodies, the detection of which (seropositivity) is an indicator of the existence of the HIV infection. 
Various treatments have been tried with various levels of success against the HIV infection, aiming at eradicating the virus or at least postponing indefinitely the onset of AIDS. Prominent among them is the treatment based on protease inhibitors, which blocks the production of infectious virions. Unfortunately, the efficiency of the drugs is thwarted by the ability of HIV to mutate rapidly. This leads to the idea of combined treatments involving more than one drug (usually protease inhibitors are combined with reverse transcription inhibitors). The idea behind this strategy is that it should take much longer for the virus to evolve to a form resistant simultaneously to more than one toxic factor. This rationale is validated by the experimental results, and the treated infected patients do indeed have extended life spans.

In what follows, we will present a simple, discrete-time model of the dynamics of Tcells and viruses under the influence of a combined treatment. Our model is inspired by a continuous-time model of Murray, which it contains at the continuous limit [8]. We start by presenting the model and then explain the physical meaning of the quantities involved:

$$
x_{n+1}=\frac{a+b x_{n}}{1+d y_{n}}, \quad y_{n+1}=f y_{n}+g z_{n}, \quad z_{n+1}=h z_{n}+k x_{n} y_{n+1},
$$

where $x, y$, and $z$ are related to the concentrations of (uninfected) T-cells ( $\mathrm{T}$ ), infectious viruses $(\mathrm{V})$, and infected T-cells (I), respectively. In order to make clear the meaning of the coefficients, we prefer to proceed to the continuous limit of (3.1) and analyze in detail the differential equations obtained. We start by putting $x=\epsilon T, y=\epsilon V$, and $z=\epsilon I$, where $\epsilon$ is a parameter which goes to 0 at the continuous limit and is related to the time step through $t=\epsilon n$. Furthermore, we take $a=\epsilon^{2} \sigma, b=(1+\epsilon \lambda)^{-1}, d=\kappa, f=(1+\epsilon \mu)^{-1}$, $g=\epsilon \eta N \nu, h=(1+\epsilon \nu)^{-1}$, and $k=\theta \kappa$, whereupon, at the limit $\epsilon \rightarrow 0$, (3.1) become

$$
\frac{d T}{d t}=\sigma-\lambda T-\kappa V T, \quad \frac{d I}{d t}=\theta \kappa V T-\nu I, \quad \frac{d V}{d t}=\eta N \nu I-\mu V .
$$

We can now comment on the significance of the various terms. In the first equation, $\sigma$ is the source of T-cells, $\lambda$ their natural death rate, and $\kappa$ the infection rate due to the presence of viruses. In the second equation, the first term describes the production of infected cells and the efficiency $\kappa$ is modulated by the action of drug therapy (reverse transcription inhibitors), where $\theta=0$ corresponds to a (theoretically) perfect drug and $\theta=1$ to no therapy at all. The second term describes the death of infected cells, with rate $\nu$. In the third equation, we have the production of viruses, $N$ viruses per bursting infected cell, modulated by the protease therapy. Again, for $\eta=0$, we have a perfect drug and for $\eta=1$ no protease inhibitor at all. The death rate of the viruses is $\mu$. This is essentially the continuous model proposed by Murray [8]. The only difference is that in Murray's model, a logistic growth of the uninfected T-cells appears. Anyway, the effect of this term is not so important, the coupling coefficient being very small. Moreover, Murray's model contains also a linear equation for noninfectious viruses. This equation is there only for bookkeeping and thus not important for our analysis. In the discussion of the stability of fixed points as well as the numerical simulations that we will present, we will use the 


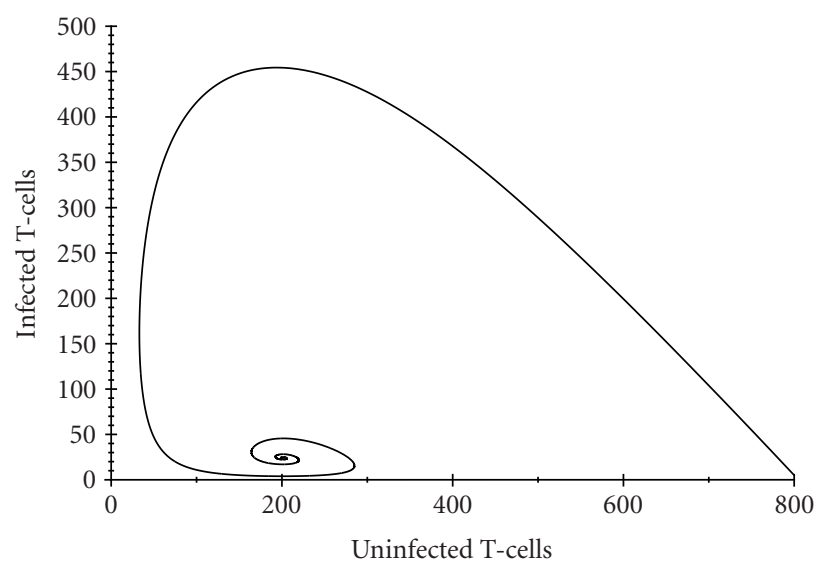

Figure 3.1. Evolution of the number of uninfected and infected T-cells in a nontherapy scenario, approximating continuous dynamics.

following set of values for the parameters entering equation (3.2). We have $\sigma=16, \lambda=$ $0.02, \kappa=3.10^{-5}, \nu=0.5, N=480$, and $\mu=3$ in the appropriate units (data adapted from references $[2,6,7,8,9,10])$.

Having explained the meaning of the parameters, we now move to the study of the equations themselves. System (3.1) possesses two fixed points. The first one, $y_{0}=z_{0}=0$, $x_{0}=a /(1-b)$, corresponds to a total absence of infection (an efficient cure). The second fixed point has $x_{1}=(1-f)(1-h) k^{-1} g^{-1}$. When we now try to compute $y_{0}$, we find that its value is positive only if $x_{1} \leq a /(1-b)$. Otherwise an unphysical (negative) value for $y_{0}$ is obtained. Thus, we have a constraint on the parameters of the mapping for the existence of this second fixed point, which, since $y_{0} z_{0} \neq 0$, corresponds to a persistent infection.

The stability of these two fixed points can be easily studied. The first fixed point is indeed stable provided $(1-f)(1-h) k^{-1} g^{-1} \geq a /(1-b)$, which is exactly the same condition we obtained for the nonexistence of a meaningful $(y \geq 0)$ second fixed point. Thus, if the quantity $(1-f)(1-h)(1-b) k^{-1} g^{-1} a^{-1}$ is larger than 1 , the no-infection fixed point is stable, otherwise a fixed point corresponding to a finite infection appears. The stability of the latter can also be easily studied. We will omit here the details of this calculation which is straightforward albeit cumbersome. For the stability of the fixed point, one requires that the characteristic polynomial have one real root smaller than one and two roots which are complex conjugate of product less than 1 . Since the parameters of the system are more or less fixed by the experimental data, it is interesting to study the behavior of the stability of the fixed point $y_{0} z_{0} \neq 0$ as a function of just $\eta \theta$ and $\epsilon$. It turns out that the stability condition can be expressed as a polynomial of degree 2 in the product $\eta \theta$ and degree 9 in $\epsilon$. For $\eta \theta=1$ (no therapy whatsoever), the only real positive root of the degree 9 polynomial (using the values of the parameters given previously in this section) is $\epsilon \approx 3.64$. When the value of $\eta \theta$ decreases, the value of this root also decreases towards a shallow minimum $\epsilon \approx 3.4365$ attained for $\eta \theta=0.1584$. Beyond this value, the critical 


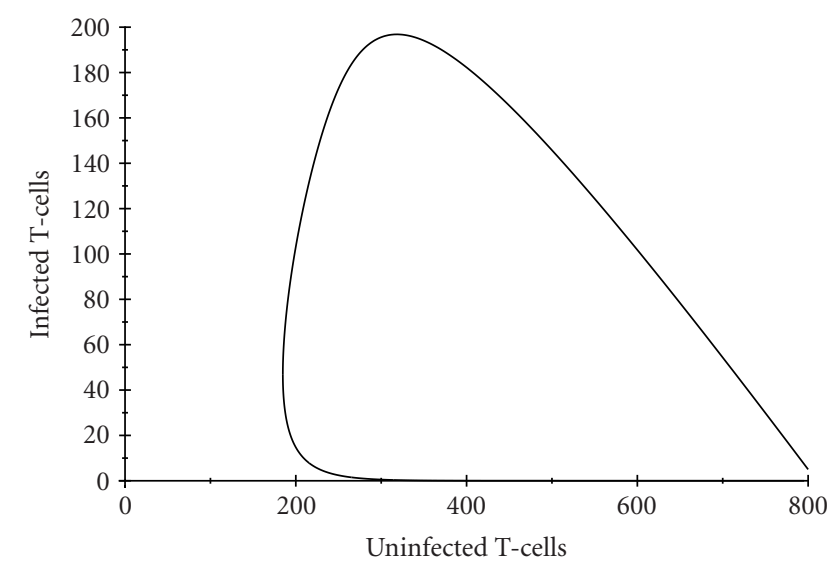

Figure 3.2. Evolution of the number of uninfected and infected T-cells in an efficient therapy scenario, approximating continuous dynamics.

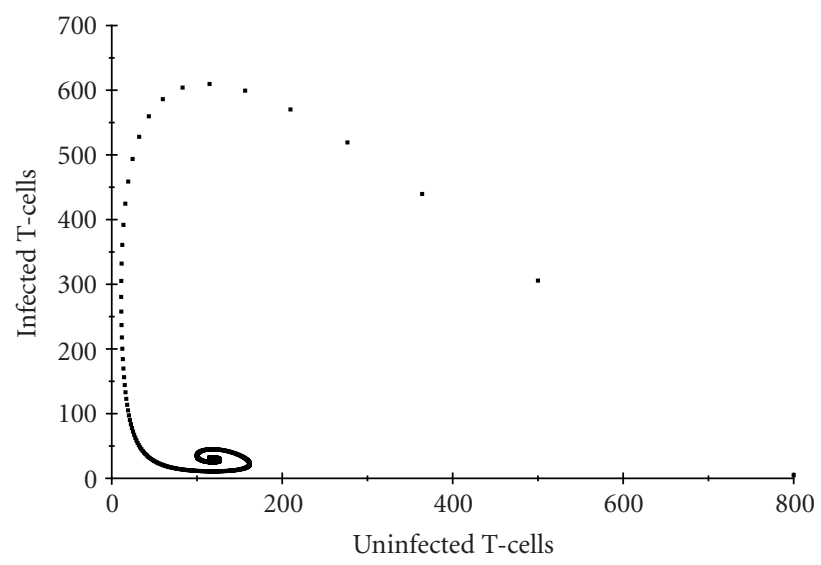

Figure 3.3. Evolution of the number of uninfected and infected T-cells in a nontherapy scenario with markedly discrete dynamics.

value of $\epsilon$ increases again. For $\epsilon$ smaller than the critical value, the fixed point is attractive. Beyond the critical value, the fixed point becomes repulsive and we have appearance of a limit cycle: the populations vary periodically with an asymptotically constant amplitude.

We have carried out numerical simulations (which are essentially the evolution of (3.1) with various initial data) for different values of the therapy-related parameters $\eta, \theta$ and also of the discretization parameter $\epsilon$. In the figures given below, we present three typical cases. Figures 3.1 and 3.2, obtained with a very small value of $\epsilon(\epsilon=0.01)$ simulate the continuous system. Two choices of $\eta \theta$ were made so as to have an ineffective treatment (in fact, no treatment at all, $\eta \theta=1)$, and an effective one $(\eta \theta=1 / 4)$. The last two figures illustrate the role of $\epsilon$ in the absence of treatment, $\eta \theta=1$. Figure 3.3 is obtained for $\epsilon=$ 0.2 , large enough for the behavior to be visibly discretized, but still well below the critical 


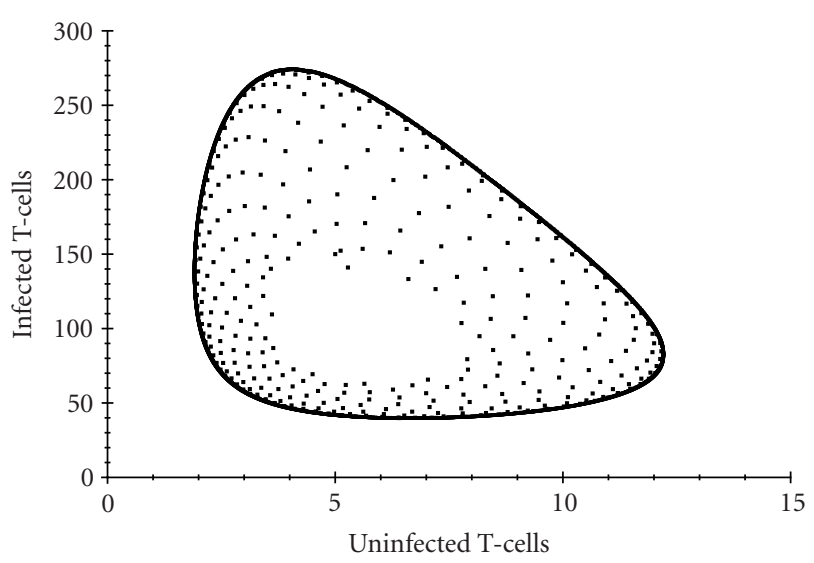

Figure 3.4. Evolution of the number of uninfected and infected T-cells in a nontherapy scenario for discrete dynamics with very large $\epsilon$.

value for limit cycle behavior. Figure 3.4 is obtained for $\epsilon=4$ beyond the critical value and displays the limit cycle behavior we predicted based on the stability analysis.

\section{Conclusion}

In this paper, we have used difference equations in order to model two problems related with AIDS. From our analysis, it is clear that the difference systems can reproduce the behavior of their continuous counterparts (an almost tautology since the difference equations contain the differential ones as continuous limits). However, the discrete systems may have surprises in store, like the existence of a limit cycle behavior for system (3.1) at large $\epsilon$.

A point we wish to stress once more in this conclusion is the interest of tailor-made discrete systems as simulators of their continuous counterparts. One can of course use, for this simulation, standard numerical algorithms, either part of some package, like Matlab or Mathematica, or directly programmed (which results in faster executions). However, we find this black-box approach somewhat unsatisfactory. Clearly, if the integration step is very small, the results of the standard numerical algorithms and of the iteration of specifically constructed discrete systems are identical. However, the latter work fine with significantly larger time steps (and thus result in much faster executions), a fact which can be of utmost importance when one simulates systems more complicated than the ones treated here. A more important point is that the discrete systems are constructed so as to preserve all the characteristics of the continuous model exactly. For instance, conserved quantities, if present, are exactly conserved and not up to some approximation related to the time step.

At a deeper level, one can ask the question of the pertinence of the discrete systems in modelling dynamical systems and, in particular, biological ones. A possible answer can be sought in the formal analogy which exists between difference and delay systems. Since the mechanisms involved have characteristic times, a description in terms of difference 
equations is most adequate (but the proper choice of the time step remains always delicate).

Concerning the epidemic insights we can draw from our model, one thing can be seen with certainty: if we have a small nucleus of seropositive individuals who may interact with healthy ones, an AIDS epidemic will invariably break out. On a predictive level, this means that only perfect screening of the total population may prevent this occurrence (a fact which is of course well known to hygiene specialists). Concerning the combineddrugs therapy of AIDS, there exist possible improvements of the model we presented here. We intend to address them in some future publication.

\section{Acknowledgment}

A. S. Carstea acknowledges a CEE scholarship through an RTN contract.

\section{References}

[1] D. J. Daley and J. Gani, Epidemic Modelling: an Introduction, Cambridge Studies in Mathematical Biology, vol. 15, Cambridge University Press, Cambridge, 1999.

[2] D. D. Ho, A. U. Neumann, A. S. Perelson, W. Chen, J. M. Leonard, and M. Markowitz, Rapid turnover of plasma virions and CD4 lymphocytes in HIV-1 infection, Nature 373 (1995), no. $6510,123-126$.

[3] W. O. Kermack and A. G. McKendrick, A contribution to the mathematical theory of epidemics, Proc. Roy. Soc. London Ser. A 115 (1927), 700-721.

[4] Contributions to the mathematical theory of epidemics, Part II, Proc. Roy. Soc. London Ser. A 138 (1932), 55-83.

[5] Contributions to the mathematical theory of epidemics, Part III, Proc. Roy. Soc. London Ser. A 141 (1933), 94-112.

[6] D. E. Kirschner and G. F. Webb, Understanding drug resistance for monotherapy treatment of HIV infection, Bull. Math. Biol. 59 (1997), no. 4, 763-785.

[7] A. R. McLean and C. A. Michie, In vivo estimates of division and death rates of human T lymphocytes, Proc. Natl. Acad. Sci. USA 92 (1995), no. 9, 3707-3711.

[8] J. D. Murray, Mathematical Biology. I. An Introduction, Interdisciplinary Applied Mathematics, vol. 17, Springer-Verlag, New York, 2002.

[9] A. S. Perelson and P. W. Nelson, Mathematical analysis of HIV-1 dynamics in vivo, SIAM Rev. 41 (1999), no. 1, 3-44.

[10] A. S. Perelson, A. U. Neumann, M. Markowitz, J. M. Leonard, and D. D. Ho, HIV-1 dynamics in vivo: virion clearance rate, infected cell life-span, and viral generation time, Science 271 (1996), no. 5255, 1582-1586.

[11] A. Ramani, A. S. Carstea, R. Willox, and B. Grammaticos, Oscillating epidemics: a discrete-time model, Phys. A 333 (2004), 278-292.

[12] T. Tokihiro, D. Takahashi, J. Matsukidaira, and J. Satsuma, From soliton equations to integrable cellular automata through a limiting procedure, Phys. Rev. Lett. 76 (1996), no. 18, 3247-3250.

[13] R. Willox, B. Grammaticos, A. S. Carstea, and A. Ramani, Epidemic dynamics: discrete-time and cellular automaton models, Phys. A 328 (2003), no. 1-2, 13-22.

K. M. Tamizhmani: Department of Mathematics, Pondicherry University, Kalapet, Pondicherry 605014, India

E-mail address: tamizh@yahoo.com 
A. Ramani: Centre de Physique Théorique, École Polytechnique, Centre National de la Recherche Scientifique (CNRS), UMR 7644, 91128 Palaiseau, France

E-mail address: ramani@cpht.polytechnique.fr

B. Grammaticos: GMPIB, Université Paris VII, case 7021, 75251 Paris, France

E-mail address: grammati@paris7.jussieu.fr

A. S. Carstea: Institute of Physics and Nuclear Engineering, Magurele, P.O. Box MG-6, Bucharest 76900, Romania

E-mail address: acarst@theory.nipne.ro 